\title{
Applications of Lie Group Analysis to Mathematical Modelling in Natural Sciences
}

\author{
N. H. Ibragimov ${ }^{1}$, R. N. Ibragimov ${ }^{2 *}$ \\ ${ }^{1}$ Department of Mathematics and ScienceBlekinge Institute of Technology \\ SE-371 79, Karlskrona, Sweden \\ 2 Department of Mathematics, University of Texas at Brownsville, TX 78520, USA
}

\begin{abstract}
Today engineering and science researchers routinely confront problems in mathematical modeling involving solutions techniques for differential equations. Sometimes these solutions can be obtained analytically by numerous traditional ad hoc methods appropriate for integrating particular types of equations. More often, however, the solutions cannot be obtained by these methods, in spite of the fact that, e.g. over 400 types of integrable second-order ordinary differential equations were summarized in voluminous catalogues. On the other hand, many mathematical models formulated in terms of nonlinear differential equations can successfully be treated and solved by Lie group methods. Lie group analysis is especially valuable in investigating nonlinear differential equations, for its algorithms act here as reliably as for linear cases. The aim of this article is, from the one hand, to provide the wide audience of researchers with the comprehensive introduction to Lie's group analysis and, from the other hand, is to illustrate the advantages of application of Lie group analysis to group theoretical modeling of internal gravity waves in stratified fluids.
\end{abstract}

Keywords and phrases: internal gravity waves, invariant solutions

Mathematics Subject Classification: 35E15

\section{Introduction}

The formulation of fundamental natural laws and of technological problems in the form of rigorous mathematical models is given frequently, even prevalently, in terms of nonlinear ordinary and partial differential equations. An appropriate method for tackling nonlinear equations is provided by Lie group analysis. Lie group analysis suggests a rigorous mathematical formulation of intuitive ideas of symmetry and provides constructive methods for solving non-linear differential equations analytically.

Just like use of microscope in biology, Lie group analysis allows to reveal symmetries of differential equations that cannot be seen otherwise.

Acquaintance with group analysis is important for constructing and investigating nonlinear mathematical models of natural and engineering problems. Numerous physical phenomena can be investigated using Lie symmetries to unearth various group invariant solutions and conservation laws that provide significant physical insight into the problem. Moreover, models of natural phenomena can often be de-

*Corresponding author. E-mail: ranis.ibragimov@utb.edu 
scribed directly in group theoretic terms. Differential equations, conservation laws, solutions to boundary value problems, and so forth can be derived from the group invariance principle.

Today, we observe the veritable explosion of advances in the field of Lie group analysis, both in applying classical and developing new methods. An extensive compilation of the results of applications of Lie group analysis to applied mathematics is given in three volumes [15]. Particularly, applications to diffusion and wave phenomena, hydrodynamics and finite-difference equations are given in Vol. 1 of [15], to industrial mathematics, field theory, nonlinear optics and acoustics, astrophysics and plasma as well as Earth sciences are summarized in Vol. 2 of [15], new trends in group analysis such as approximate symmetries of equations with a small parameter and extension of the group analysis to the space of distributions as well as computational methods are presented in Vol. 3 of [15]. Applications of Lie group analysis to Riemann spaces is provided in [14].

The aim of this article is to impart to the wide audience of researchers and students several recent results in this area whose discussion discloses the advantages to be gained from the use of the group theoretic approach. The emphasis will be on an application of Lie group analysis to mathematical modelling of internal gravity waves in the ocean.

\section{Preliminaries on symmetry groups and invariant solutions}

\subsection{Symmetry groups and invariant solutions}

Let $x=\left(x^{1}, \ldots, x^{n}\right)$ and $u=\left(u^{1}, \ldots, u^{m}\right)$ be independent and dependent variables, respectively. We will deal with one-parameter groups $G$ of transformations of the form

$$
\begin{array}{ll}
\bar{x}^{i}=\varphi^{i}(x, a), & \left.\varphi^{i}\right|_{a=0}=x^{i}, \\
\bar{u}^{\alpha}=\psi^{\alpha}(x, u, a), & \left.\psi^{\alpha}\right|_{a=0}=u^{\alpha},
\end{array}
$$

depending on a continuous parameter $a$. The infinitesimal transformations of the group $G$ are written

$$
\bar{x}^{i} \approx x^{i}+a \xi^{i}(x), \quad \bar{u}^{\alpha} \approx u^{\alpha}+a \eta^{\alpha}(x, u) .
$$

The generator of the group $G$ is the linear first-order differential operator

$$
X=\xi^{i}(x) \frac{\partial}{\partial x^{i}}+\eta^{\alpha}(x, u) \frac{\partial}{\partial u^{\alpha}} .
$$

We employ the usual convention on summation over repeated indices.

Let us consider a (linear or nonlinear) system of partial differential equations

$$
F_{\alpha}\left(x, u, u_{(1)}, \ldots, u_{(s)}\right)=0, \quad \alpha=1, \ldots, m,
$$

where $u_{(1)}, \ldots, u_{(s)}$ denote the sets of partial derivatives of the respective orders, e.g. $u_{(1)}=\left\{u_{i}^{\alpha}\right\}, u_{(2)}=$ $\left\{u_{i j}^{\alpha}\right\}$ with

$$
u_{i}^{\alpha}=\frac{\partial u^{\alpha}(x)}{\partial x^{i}}, \quad u_{i j}^{\alpha}=\frac{\partial^{2} u^{\alpha}(x)}{\partial x^{i} \partial x^{i}} .
$$

The group $G$ is called a symmetry group (or admitted group) for Eqs. (2.3) if the system (2.3) has the same form when it is rewritten in the new variables $\bar{x}, \bar{u}$ :

$$
F_{\alpha}\left(\bar{x}, \bar{u}, \bar{u}_{(1)}, \ldots, \bar{u}_{(s)}\right)=0, \quad \alpha=1, \ldots, m .
$$

The generator of the symmetry group $G$ is called an admitted operator or an infinitesimal symmetry for Eqs. (2.3). The effective way for constructing the symmetry group for Eqs. (2.3) is to solve the determining equations 


$$
\left.X_{(s)}\left(F_{\alpha} x, u, u_{(1), \ldots,} u_{(s)}\right)\right|_{(2.3)}=0, \quad \alpha=1, \ldots, m,
$$

for the generator (2.2) of the symmetry group $G$, where $X_{(s)}$ is the $s^{\text {th }}$ prolongation for the generator $X$ of the group $G$, and the notation $\left.\right|_{(2.3)}$ means evaluated on Eqs. (2.3) (see e.g., [39], [38], [13], [22] for the nomenclature).

The admitted group $G$ maps any solution of the system (2.3) into a solution of the same system. A solution that is mapped by $G$ into itself is known as an invariant solution with respect to the group $G$. The invariant solutions for a group with a generator $X$ are obtained as follows (see, e.g. [13]). One calculates the invariants $J(x, u)$ of the group $G$ by solving the first-order linear partial differential equation

$$
X(J) \equiv \xi^{i}(x) \frac{\partial J}{\partial x^{i}}+\eta^{\alpha}(x, u) \frac{\partial J}{\partial u^{\alpha}}=0,
$$

or its characteristic system

$$
\frac{d x^{1}}{\xi^{1}(x)}=\cdots=\frac{d x^{n}}{\xi^{n}(x)}=\frac{d u^{1}}{\eta^{1}(x, u)}=\cdots=\frac{d u^{m}}{\eta^{m}(x, u)} .
$$

It is manifest from Eqs. (2.6) that the group has $n+m-1$ functionally independent invariants of the following form:

$$
\lambda_{1}(x), \ldots, \lambda_{n-1}(x), \quad \Phi_{1}(x, u), \ldots, \Phi_{m}(x, u) .
$$

Then we let

$$
\Phi_{\alpha}=\phi_{\alpha}\left(\lambda_{1}, \ldots, \lambda_{n-1}\right)
$$

solve these equations for $u^{1}, \ldots, u^{m}$, substitute the resulting expressions for $u$ in Eqs. (2.3) and obtain a system of partial differential equations for the unknown functions $\phi_{\alpha}\left(\lambda_{1}, \ldots, \lambda_{n-1}\right)$ depending of $n-1$ variables.

If we will require the invariance with respect to a symmetry group with $n-1$ linearly independent generators, then we will have only one invariant of the form $\lambda(x)$, and the system (2.3) will be reduced to ordinary differential equations for unknown functions $\phi \alpha(\lambda)$.

\section{Adjoint and self-adjoint equations to nonlinear equations}

In terms of group theoretical modeling, the dynamics of the system in the question can often be described by means of the formal Lagrangian and the adjoint equations that has been intorduced originally in Ibragimov, [16]. In the case of the system (2.3), the formal Lagrangian has the form

$$
\mathcal{L}=\mu^{\beta} F_{\beta}\left(x, u, u_{(1)}, \ldots, u_{(s)}\right)
$$

and the adjoint equations to Eqs. (2.3) are defined by

$$
F_{\alpha}^{*}\left(x, u, \mu, u_{(1)}, \mu_{(1)}, \ldots, u_{(s)}, \mu_{(s)}\right)=0, \quad \alpha=1, \ldots, m .
$$

Here $\mu=\left(\mu^{1}, \ldots, \mu^{m}\right)$ are new dependent variables and $F_{\alpha}^{*}$ are given by

$$
F_{\alpha}^{*}\left(x, u, \mu, u_{(1)}, \mu_{(1)}, \ldots, u_{(s)}, \mu_{(s)}\right)=\frac{\delta \mathcal{L}}{\delta u^{\alpha}},
$$

where

$$
\frac{\delta}{\delta u^{\alpha}}=\frac{\partial}{\partial u^{\alpha}}-D_{i} \frac{\partial}{\partial u_{i}^{\alpha}}+D_{i} D_{j} \frac{\partial}{\partial u_{i j}^{\alpha}}-D_{i} D_{j} D_{k} \frac{\partial}{\partial u_{i j k}^{\alpha}}+\cdots
$$

denote the variational derivatives and

$$
D_{i}=\frac{\partial}{\partial x^{i}}+u_{i}^{\alpha} \frac{\partial}{\partial u^{\alpha}}+\mu_{i}^{\alpha} \frac{\partial}{\partial \mu^{\alpha}}+u_{i j}^{\alpha} \frac{\partial}{\partial u_{j}^{\alpha}}+\mu_{i j}^{\alpha} \frac{\partial}{\partial \mu_{j}^{\alpha}}+u_{i j k}^{\alpha} \frac{\partial}{\partial u_{j k}^{\alpha}}+\cdots
$$


is the operator of total differentiation with respect to $x^{i}$. In the case of linear equations this definition is equivalent to the classical definition of the adjoint equations.

Eqs. (2.3) are said to be self-adjoint if the adjoint system (3.2) becomes equivalent to the original system (2.3) upon a substitution

$$
\mu^{\alpha}=h^{\alpha}(u), \quad \alpha=1, \ldots, m,
$$

where not all $h^{\alpha}(u)$ are constants. If all $h^{\alpha}(u)$ are constants we have the singular case of self-adjointness. As will be shown in sequel, the nonlinear system describing the dynamics of internal waves in the ocean has a remarkable property to be self-adjoint. This property is crucial for constructing conservation laws provided in the present study.

\subsection{Conservation laws for self-adjoint equations}

The new conservation laws for internal gravity waves reported here were deduced on the basis of the new approach for constructing conservation laws for self-adjoint nonlinear systems. We will apply this approach to a self-adjoint system $(2.3)$ of the third-order $(s=3)$. In this case, the general conservation theorem demonstrated in [16] shows that if Eqs. (2.3) admit the group with the generator (2.2) then one can obtain the conservation law $D_{i}\left(C^{i}\right)=0$ for Eqs. (2.3) by using the formula

$$
\begin{aligned}
& C^{i}=\xi^{i} \mathcal{L}+W^{\alpha}\left[\frac{\partial \mathcal{L}}{\partial u_{i}^{\alpha}}-D_{j}\left(\frac{\partial \mathcal{L}}{\partial u_{i j}^{\alpha}}\right)+D_{j} D_{k}\left(\frac{\partial \mathcal{L}}{\partial u_{i j k}^{\alpha}}\right)\right] \\
& +D_{j}\left(W^{\alpha}\right)\left[\frac{\partial \mathcal{L}}{\partial u_{i j}^{\alpha}}-D_{k}\left(\frac{\partial \mathcal{L}}{\partial u_{i j k}^{\alpha}}\right)\right]+D_{j} D_{k}\left(W^{\alpha}\right)\left[\frac{\partial \mathcal{L}}{\partial u_{i j k}^{\alpha}}\right],
\end{aligned}
$$

where $\mathcal{L}$ is given by (3.1), $W^{\alpha}=\eta^{\alpha}-\xi^{j} u_{j}^{\alpha}$ and the additional variables $\mu^{\alpha}$ are eliminated by using Eqs. $(3.5)$.

\section{Group theoretic modelling}

\subsection{Determination of linear heat diffusion by Galilean group}

It has been shown in Ref. [17] that the Galilean group determines the linear heat diffusion with an arbitrary initial distribution of the temperature field without using Fourier's law.

A sketch of the construction is as follows. The Galilean group with $n$ spatial variables $x^{i}$, time $t$ and the dependent variable $u$ has the generators

$$
\begin{gathered}
X_{0}=\frac{\partial}{\partial t}, \quad X_{i}=\frac{\partial}{\partial x^{i}}, \quad X_{i j}=x^{j} \frac{\partial}{\partial x^{i}}-x^{i} \frac{\partial}{\partial x^{j}} \quad(i<j), \\
Y_{i}=t \frac{\partial}{\partial x^{i}}, \quad T_{1}=u \frac{\partial}{\partial u}, \quad i, j=1, \ldots, n .
\end{gathered}
$$

The operators (4.1) generate the Euclidean group (translations and rotations in the $x$-space) augmented by the time translation generated by $X_{0}$, the operators $Y_{i}$ in (4.2) generate the Galilean transformations, whereas the generator $T_{1}$ of the scaling transformation is responsible for the homogeneity in the $u$-space.

One considers the semi-scalar representation of the Galilean group obtained by a linear prolongation of the action of the Galilean transformation to the dependent variable, i.e. by letting

$$
Y_{i}=t \frac{\partial}{\partial x^{i}}+\sigma^{i}(t, x) u \frac{\partial}{\partial u}, \quad i=1, \ldots, n,
$$

where not all $\sigma^{i}(t, x)$ are identically zero. One can prove that this representation is unique, namely $\sigma^{i}=-x^{i}$. Hence, the generators of the semi-scalar representation of the Galilean group comprise the operators (4.1) and

$$
Y_{i}=t \frac{\partial}{\partial x^{i}}-x^{i} u \frac{\partial}{\partial u}, \quad T_{1}=u \frac{\partial}{\partial u} .
$$


Furthermore, the obtained semi-scalar representation of the Galilean group admits the unique extension by scaling transformations. It has the generator

$$
T_{2}=2 t \frac{\partial}{\partial t}+x^{i} \frac{\partial}{\partial x^{i}} .
$$

Thus, the maximal extended semi-scalar representation of the Galilean group is generated by the operators (4.1), (4.3) and (4.4). Finally, the invariance with respect to this extended semi-scalar representation of the Galilean group determines uniquely the fundamental solution

$$
E(t, x)=(2 \sqrt{\pi t})^{-n} \mathrm{e}^{-r^{2} /(4 t)}, \quad r^{2}=|x|^{2}=\left(x^{1}\right)^{2}+\cdots+\left(x^{n}\right)^{2},
$$

and hence Poisson's formula

$$
u(t, x)=\frac{1}{(2 \sqrt{\pi t})^{n}} \int_{\mathbf{R}^{n}} u_{0}(y) \mathrm{e}^{-|x-y|^{2} /(4 t)} d y, \quad t>0,
$$

for the diffusion of an arbitrary initial temperature $u_{0}(y)$.

\subsection{Propagation of light in curved space-times}

One of remarkable properties of the classical wave equation

$$
u_{t t}-u_{x x}-u_{y y}-u_{z z}=0
$$

is existence of a sharp rear front of waves. It means that the light waves propagate without diffusion. This property is known as the Huygens principle in the sense of Hadamard's "minor premise". Hadamard [11] (see also [12]) posed the problem of describing all linear second-order hyperbolic equations

$$
g^{i j}(x) u_{i j}+b^{i}(x) u_{i}+c(x) u=0
$$

satisfying the Huygens principle. In general, it is still an open problem. However, Hadamard's problem has been completely solved (see [18]), using Lie group analysis, in the case of four independent variables $x=\left(x^{1}, \ldots, x^{4}\right)$ under the assumption that the space-time $V_{4}$ associated with Eq. (4.5), i.e. the Riemannian space with the metric

$$
d s^{2}=g_{i j}(x) d x^{i} d x^{j}, \quad g_{i j}(x) g^{i k}(x)=\delta_{i}^{k},
$$

has a nontrivial conformal group. We give here a brief description of this result.

The wave equation in curved space times with nontrivial conformal group can be reduced by appropriate conformal transformations and changes of coordinates to the following form

$$
u_{t t}-u_{x x}-f(x-t) u_{y y}-2 \varphi(x-t) u_{y z}-u_{z z}=0,
$$

where $f$ and $\varphi$ are arbitrary functions of one variable satisfying the condition $f(x-t)-\varphi^{2}(x-t)>0$ which guarantees that Eq. (4.6) is hyperbolic. Validity of the Huygens principle is manifest from the explicit form of the solution of Cauchy's problem

$$
t=0: u=g(x, y, z), u_{t}=h(x, y, z)
$$

for Eq. (4.6). The solution is obtained in [18] in the integral representation similar to Poisson's formula for the wave equation. It has the form

$$
u=T\left[h-g_{x}\right]+\left(\frac{\partial}{\partial t}+\frac{\partial}{\partial x}\right) T[g] .
$$


Here, e.g.

$$
T[g](t, x, y, z)=\frac{1}{4 \pi} \int_{x-t}^{x+t} d \xi \int_{0}^{2 \pi} g(\xi, y+A \cos \theta, z+B \cos \theta+C \sin \theta) d \theta
$$

with

$$
\begin{gathered}
A=\sqrt{(x+t-\xi)[F(\xi)-F(x-t)]} \\
B=\frac{x+t-\xi}{A}[\Phi(\xi)-\Phi(x-t)], \quad C=\sqrt{t^{2}-(x-\xi)^{2}-B^{2}}
\end{gathered}
$$

where $F(\sigma)=\int f(\sigma) d \sigma, \quad \Phi(\sigma)=\int \varphi(\sigma) d \sigma$.

\subsection{Group analysis of Moffatt's model for liquid metal systems}

Interest to applications of Magnetohydrodynamics in metallurgical industry in 1970s has been motivated by possibilities of using alternating magnetic fields in processing of liquid metals when high quality of the product is required. High frequency external magnetic fields are used in casting process in the steel industry in order to control a flow of liquid metals and to generate internal stirring within the liquid phase. This allows one to reach homogeneity of solidifying metals by eliminating blowholes caused by escaping gases.

Mathematical model of "skin effects" caused by an electromagnetic field in a thin surface layer of liquid metals has been suggested by Moffatt [35]. He uses boundary layer equations for the stream function $\psi(x, y)$ :

$$
\psi_{y} \psi_{x y}-\psi_{x} \psi_{y y}=\nu \psi_{y y y}, \quad \nu=\text { const. }
$$

The physical arguments discussed in Moffatt [35] yield the boundary conditions

$$
\begin{aligned}
& \psi=0, \quad \psi_{y}=A x^{m} \quad \text { on } \quad y=0, \\
& \psi_{y} \rightarrow 0 \quad \text { as } \quad y \rightarrow \infty .
\end{aligned}
$$

and show that

$$
\begin{array}{lll}
A<0 & \text { when } m+1<0 & \text { (near a sharp corner) } \\
A>0 & \text { when } m+1>0 & \text { (far from the corner) }
\end{array}
$$

The application of the invariance principle to Eq. (4.8) shows [19] that it admits the infinite group with the generators

$$
\begin{gathered}
X_{1}=x \frac{\partial}{\partial x}+\psi \frac{\partial}{\partial \psi}, \quad X_{2}=y \frac{\partial}{\partial y}-\psi \frac{\partial}{\partial \psi}, \\
X_{3}=\frac{\partial}{\partial x}, \quad X_{4}=\frac{\partial}{\partial \psi}, \quad X_{5}=h(x) \frac{\partial}{\partial y}
\end{gathered}
$$

and that the requirement of invariance of the boundary conditions (4.9) singles out the one-parameter subgroup generated by the operator $X=2 X_{1}+(1-m) X_{2}$. According to the invariance principle, the solution of the boundary value problem (4.8)-(4.9) should be the invariant solution with respect to $X$. In this case Eqs. (2.7)-(2.8) for the invariant solution yield

$$
\psi=\left(\nu|A| x^{m+1}\right)^{1 / 2} f(\lambda), \quad \lambda=\left(\nu^{-1}|A| x^{m-1}\right)^{1 / 2} y .
$$

The invariant form (4.11) simplifies the boundary-value problem (4.8)-(4.9) significantly by reducing it to the following boundary-value problem for an ordinary differential equation:

$$
f^{\prime \prime \prime}+\frac{m+1}{2} f f^{\prime \prime}-m f^{\prime 2}=0
$$




$$
f(0)=0, \quad f^{\prime}(\infty)=0, \quad f^{\prime}(0)=\left\{\begin{array}{l}
+1 \text { when } m+1>0 \\
-1 \text { when } m+1<0
\end{array}\right.
$$

Furthermore, the reckoning shows that Eq. (4.8) is self-adjoint. Application of Eqs. (3.6) to the symmetries (4.10) gives two conservation laws for Eq. (4.8):

$$
\begin{gathered}
D_{x}\left(\psi_{y}^{2}\right)+D_{y}\left(-\psi_{x} \psi_{y}-\nu \psi_{y y}\right)=0 \\
D_{x}\left(\psi \psi_{y}^{2}\right)+D_{y}\left(\frac{\nu}{2} \psi_{y}^{2}-\psi \psi_{x} \psi_{y}-\nu \psi \psi_{y y}\right)=0 .
\end{gathered}
$$

The ordinary differential equation (4.12) and the corresponding boundary conditions are presented in [35] but the substitution (4.11) has been misprinted there. The conservation laws (4.13) have been obtained recently [20].

\subsection{Internal gravity wave beams in the deep ocean}

One of the main reasons for studying internal waves remains the fact that they are suspected to play an important role in the dynamics of the ocean, especially in affecting the large-scale general circulation model (see e.g., [36], [49]). Furthermore, mutual interactions between internal waves produce mixing in the interior of the ocean providing an important link in the presumed energy cascade from large to small scales (e.g.,[40], [47]). One of the practical needs to better understand mixing processes in the ocean resides in the fact that mixing plays an important role in maintaining a gradual transition between the sun-warmed surface layer of the ocean and the upwelling cold, dense water formed at high latitudes (e.g., [49], [43], [29], [8]). The subject of mixing processes due to breaking of internal waves in the ocean and atmosphere has received much attention in recent years (see e.g.[48] for the review).

In this paper, we address the nonlinear equations of motion for internal waves affected by the earth's rotation. The axes are $x$ (assumed eastward for definiteness) $y$ (northward) and $z$ vertically upward. The fluid velocity is $\vec{u}=(u, v, w)$ relative to $(x, y, z)$. The ocean has a uniform mean vertical stratification $\bar{\rho}(z)$, where $\rho$ represents density. In two dimensions, the unforced non-dissipative Boussinesq nonhydrostatic equations of motion are written as

$$
\begin{aligned}
u_{t}+u u_{x}+w u_{z}-f v & =-p_{x}, \\
v_{t}+u v_{x}+w v_{z}+f u & =0, \\
w_{t}+u w_{x}+w w_{z} & =-\rho g-p_{z}, \\
\rho_{t}+u \rho_{x}+w \rho_{z}-\frac{N^{2}}{g} w & =0, \\
u_{x}+w_{z} & =0 .
\end{aligned}
$$

Here suffices denote differentiation, $f$ is the inertial frequency which depends on the rotation rate of the earth (angular velocity $\Omega=2 \pi \mathrm{rad} /$ day $\approx 0.73 \times 10^{-4} \mathrm{~s}^{-1}$ ), $g$ is the acceleration due to gravity, $p$ is the pressure, so that $p$ and $\rho$ are to be interpreted as the pressure and density departures from their mean state

$$
\bar{\rho}(z)=-\frac{\rho_{0}}{g} N^{2} z, \quad \bar{p}(z)=p_{0}-\rho_{0} g z-g \int_{0}^{z} \bar{\rho}(\xi) d \xi,
$$

in which $\rho_{0}$ is the constant reference density, $N$ is the buoyancy frequency defined by

$$
N^{2}=-\frac{g}{\rho_{0}} \frac{d \bar{\rho}}{d z}
$$

and we require $\rho_{0}+\bar{\rho}$ and $\bar{p}$ to be consistent with the state of rest, i.e.,

$$
\frac{d \bar{p}}{d z}=-\left(\rho_{0}+\bar{\rho}\right) g
$$


The quantity $N$ measures the degree of density stratification of a fluid with average potential density $\rho(z)$ and thus represents the frequency with which a vertically displaced fluid element would be expected to oscillate because of restoring buoyancy forces. If the displacement is not strictly vertical, as in the case for internal waves, the restoring force is less so the frequency of oscillations is reduced. In the mid/high latitude upper ocean, $N$ is typically one or two orders of magnitude larger than $f\left(N \sim 10^{-3}\right.$ to $10^{-2}$ $\left.\mathrm{s}^{-1} ; f \sim 10^{-4} \mathrm{~s}^{-1}\right)$. Our model is idealized by assuming $N$ to be uniform over the extent of the fluid. This corresponds to a vertically linear density variation. While this simplification is commonly used in laboratory and theoretical studies and it is quite reasonable for the thermocline region, it is not common in the deep region of the ocean with the exception when considering short wavelengths in comparison with the scale of density changes ([43]). At low frequencies, close to $f$, rotational effects are important. Such internal waves are sometimes called inertial-internal waves. At high frequencies, close to $N$ and far from $f$, rotational effects are negligible.

From the analytical standpoint, internal waves are often considered as monochromatic plane waves [32] while oceanic measurements usually report a beam-like structure with a width smaller than a wavelength (see e.g. [33]). The latter observations are attributed to the fact that the propagation of internal gravity waves emitted from a localized source is anisotropic (the frequency of internal waves is independent on the magnitude of wave number vector so that any sinusoidal plane-wave disturbances in a uniformly stratified Boussinesq fluid obeying the linear dispersion relation satisfy the nonlinear equations of motion) and so it is described by beams along which the energy of internal waves is usually concentrated. This fact makes it possible to construct more general class of disturbances which represent wave beams propagating in the direction transverse to the general uniform plane wave profile.

In view of the anisotropic nature of internal gravity waves, the nonlinear model (4.14) - (4.18) is constrained by the dispersion relation

$$
\omega^{2}=\frac{N^{2} k^{2}+f^{2} m^{2}}{k^{2}+m^{2}}
$$

meaning that the frequency $\omega$ of internal waves depends only on the orientation of the wavenumber vector $\vec{k}=(k, m)$ but not its magnitude. The anisotropic property $(4.22)$ can also be written in the trigonometric form

$$
\omega^{2}=N^{2} \sin ^{2} \alpha+f^{2} \cos ^{2} \alpha,
$$

$\alpha$ being the beam inclination relative to the horizontal.

We consider the model under "symmetric" conditions where all dynamical fields are independent of $y$, so that all $y$ derivatives drop out. In light of the nondivergent nature of the velocity field in the $x-z$ plane, it is convenient to recast the model (4.14) - (4.18) by introducing a stream function $\psi$ via $u=\psi_{z}, w=-\psi_{x}$. This allows one to exclude the pressure and to write the governing equations in the form

$$
\begin{gathered}
\Delta_{2} \psi_{t}-g \rho_{x}-f v_{z}=J\left(\psi, \Delta_{2} \psi\right), \\
v_{t}+f \psi_{z}=J(\psi, v), \\
\rho_{t}+\frac{N^{2}}{g} \psi_{x}=J(\psi, \rho),
\end{gathered}
$$

where $\Delta_{2}$ denotes the two-dimensional (in $x, z$ ) Laplacian and the nonlinear advective terms are written by means of the Jacobian operator $J(a, b)=a_{x} b_{z}-a_{z} b_{x}$, in which subscripts denote partial differentiation.

Some particular class of invariant solutions can be deduced on the basis of the obvious symmetries of Eqs. (4.24)-(4.26), namely of the translational symmetries

$$
X_{1}=\frac{\partial}{\partial t}, \quad X_{2}=\frac{\partial}{\partial x}, \quad X_{3}=\frac{\partial}{\partial z},
$$


and the following dilation generator

$$
X_{4}=x \frac{\partial}{\partial x}+z \frac{\partial}{\partial z}+v \frac{\partial}{\partial v}+\rho \frac{\partial}{\partial \rho}+2 \psi \frac{\partial}{\partial \psi} .
$$

It was shown in our previous work [23] that uni-directional internal wave beams, i.e., the nonlinear solution obeying the dispersion relation (4.23), can also be obtained as invariant solutions of nonlinear equations of motion. Namely, the invariant solution based on the dilation and translation transformations can be represented by uni-directional internal wave beams of the form

$$
\psi(\eta, t)=e^{-i \omega t} \int_{-\infty}^{0} \widehat{Q}(s) e^{i s \eta} d s+e^{i \omega t} \int_{0}^{\infty} \widehat{Q}^{*}(s) e^{-i s \eta} d s
$$

where the asterisk * denotes the complex conjugate and $\eta=k x+m z=x \sin \alpha-z \cos \alpha$ is the invariant of the translational symmetries. In our notation $\widehat{Q}(s)=\int_{-\infty}^{\infty} Q(\eta) e^{-i s \eta} d \eta$ is the Fourier transform of the initial condition for $\psi(\eta, 0)$ which represents an amount of energy radiated along the internal planewave beam at the angle $\alpha$ in the positive or negative (depending on the sign of $s$ ) direction. As has been justified in [45] and illustrated in our previous work [23], the anisotropic property expressed (4.22) makes it possible to construct, via superposition of sinusoidal plane waves with wavenumbers inclined at the same angle $\alpha$ to the vertical, general plane-wave disturbances of frequency $\omega$ in the form of beams, that are uniform along the transverse direction $\xi=x \cos \alpha+z \sin \alpha$ and have general profile along the invariant $\eta$. Furthermore, since $Q(\eta)$ is an arbitrary wave amplitude of a superposition of plane waves, the direction of the beam propagation satisfying the initial condition $\psi(\eta, 0)=Q(\eta)$ is determined uniquely. Namely, since for any sinusoidal wave of the form $h(x)=a \sin (k x-\omega t)$ the phase velocity $\vec{c}$ can be written as $\left(\omega / k^{2}\right) \vec{k}$, the sign of $s$ in formula (4.27) is determined uniquely by the sign of the frequency. Particularly, the direction of energy propagation is along the group velocity $\vec{c}_{g}=\nabla_{\vec{k}} \omega$ which, according to the dispersion relation (4.23), is orthogonal to the phase velocity $\vec{c}$ so that $\vec{c}_{g}$ and $\vec{c}$ have opposite vertical components. While the invariant solution (4.27) describes plane-wave beams for any choice of the amplitude $Q(\eta)$, uni-directional beams, in which energy propagates in one direction, involve plane waves with wavenumbers of the same sign only. For example, in view of four possible configurations of the group $\vec{c}_{g}$ and phase $\vec{c}$ velocities, for the energy to be radiated upward to the right, the radiation condition requires that $k>0$ and $m<0$, (i.e., the wavenumber vector $\vec{k}$ is along $\eta$ and thus directed downward to the right) and so forth. The analytic examples showing that the obtained generalized invariant solution (4.27) represents the uni-directional internal plane-wave beams satisfying the radiation condition (i.e., propagating in the interior of the ocean in a preferred direction away from the source) have been considered in our earlier work in Ref. [23].

We point out that the invariant solution (4.27) can also be deduced from the anisotropic property of internal waves as linear sinusoidal wavetrains satisfying the linear approximation

$$
\Delta \psi_{t t}+N^{2} \psi_{x x}+f^{2} \psi_{z z}=0
$$

of the model (4.24) - (4.26) (see e.g.[44]). However, most general forms of exact solutions for the model (4.24) - (4.26) can not be guessed from the anisotropic property. On the other hand the most general forms of exact solutions can be deduced on a regular basis, i.e., by integrating the given model in question by means of Lie group analysis.

Solution of the determining equations shows that the maximal symmetry group for the model (4.24) (4.26) with $f \neq 0$ is generated by the infinite-dimensional Lie algebra spanned by the following operators:

$$
X_{1}=\frac{\partial}{\partial v}, \quad X_{2}=\frac{\partial}{\partial \rho}, \quad X_{a}=a(t) \frac{\partial}{\partial \psi}, \quad X_{4}=\frac{\partial}{\partial t},
$$




$$
\begin{gathered}
X_{b}=b(t)\left[\frac{\partial}{\partial x}-f \frac{\partial}{\partial v}\right]+b^{\prime}(t) z \frac{\partial}{\partial \psi}, \quad X_{c}=c(t)\left[\frac{\partial}{\partial z}+\frac{N^{2}}{g} \frac{\partial}{\partial \rho}\right]-c^{\prime}(t) x \frac{\partial}{\partial \psi}, \\
X_{7}=x \frac{\partial}{\partial x}+z \frac{\partial}{\partial z}+v \frac{\partial}{\partial v}+\rho \frac{\partial}{\partial \rho}+2 \psi \frac{\partial}{\partial \psi}, \\
X_{8}=t \frac{\partial}{\partial t}+2 x \frac{\partial}{\partial x}+2 z \frac{\partial}{\partial z}+3 \psi \frac{\partial}{\partial \psi}-2 f x \frac{\partial}{\partial v}+2 \frac{N^{2}}{g} z \frac{\partial}{\partial \rho}, \\
X_{9}=z \frac{\partial}{\partial x}-x \frac{\partial}{\partial z}-\frac{1}{f}\left[g \rho+\left(f^{2}-N^{2}\right) z\right] \frac{\partial}{\partial v}+\frac{1}{g}\left[f v+\left(f^{2}-N^{2}\right) x\right] \frac{\partial}{\partial \rho} .
\end{gathered}
$$

Here $a(t), b(t)$ and $c(t)$ are arbitrary functions of time $t$. The wide class of exact solutions is represented implicitly by the Lie algebra (4.29) - (4.33). For example, using the rotational symmetry $X_{9}(4.33)$ and scaling symmetry $X_{7}(4.31)$ we obtain the invariant solution

$$
\begin{gathered}
v=\frac{1}{f}\left[\left(2 \phi^{2}(t)+A+\frac{N^{2}-f^{2}}{2}\right) x+2 \phi^{\prime}(t) z\right], \\
\rho=\frac{1}{g}\left[2 \phi^{\prime 2}(t) x-\left(2 \phi^{2}(t)+A-\frac{N^{2}-f^{2}}{2}\right) z\right], \\
\psi=\left(x^{2}+z^{2}\right) \phi(t), \quad A=\text { const. }
\end{gathered}
$$

where $\phi(t)$ is defined by the differential equation

$$
\phi^{\prime \prime 3}+\left(A+\frac{f^{2}+N^{2}}{2}\right) \phi=0 .
$$

Preliminary Lie group analysis of the rotationally invariant solution (4.34) - (4.36) for internal gravity waves in the ocean, namely the analytic and numerical comparison with the linear approximation and experimental results on internal wave beams generated from a vertically oscillating sphere studied in [7], can also be found in our pre-print [21]. The similarity to the 3D case, in which mechanical oscillators act as point sources rather than as line sources (and so the generated gravity wave disturbances have a conical shape, containing almost perfectly circular phase lines in planes of constant height) can be found in our earlier work [28], where such gravity waves were linked to spinning patterns in the atmosphere.

The formal Lagrangian (3.1) for Eqs. (4.24) - (4.26) is written

$$
\mathcal{L}=\varphi\left[\Delta_{2} \psi-g \rho_{x}-f v_{z}-J\left(\psi, \Delta_{2} \psi\right)\right]+\varphi\left[v_{t}+f \psi_{z}-J(\psi, v)\right]+r\left[\rho_{t}+\frac{N^{2}}{g} \psi_{x}-J(\psi, \rho)\right]
$$

where $\varphi, \mu$ and $r$ are new dependent variables. The adjoint equations (3.3) to Eqs. (4.24) - (4.26) are obtained by taking the variational derivatives $(3.4)$ of $\mathcal{L}$. The latter procedure shows that the adjoint equations (3.3) coincide with Eqs. (4.24) - (4.26) after the following substitution (3.5):

$$
\varphi=\psi, \quad \mu=-v, \quad r=-\frac{g^{2}}{N^{2}} \rho .
$$

Thus the system (4.24) - (4.26) is self-adjoint.

Applying the conservation formulae (3.6) to the symmetries (4.29) - (4.33) and using the substitution (4.39) we obtain the differential conservations equations

$$
D_{t}\left(C^{1}\right)+D_{x}\left(C^{2}\right)+D_{z}\left(C^{3}\right)=0 .
$$

They can be written equivalently in the integral form 


$$
\begin{gathered}
\frac{d}{d t} \iint v d x d z=0, \quad \frac{d}{d t} \iint \rho d x d z=0, \quad \frac{d}{d t} \iint\left[v^{2}+\frac{g^{2}}{N^{2}} \rho^{2}+|\nabla \psi|^{2}\right] d x d z=0 \\
\frac{d}{d t} \iint\left[f x v-g z \rho-\frac{1}{2}|\nabla \psi|^{2}\right] d x d z=0, \quad \frac{d}{d t} \iint\left[v \rho+f x \rho-\frac{N^{2}}{g} z v\right] d x d z=0 .
\end{gathered}
$$

We remark that similar conservation laws were also obtained in [1] in which the classical and nonlinear stability of baroclinic jets was compared to symmetric disturbances for nonhydrostatic, adiabatic, Boussinesq equations. Within the context of the Fjortoft's theorem on an energy-Casimir (or pseudoenergy) stability analysis studied in [6], we associate the first conservation law in (4.41) with a Hamiltonian, which differs from the energy by a Casimir invariants which constitute "hidden" conservation laws of the Eulerian representation of fluid flows. Thus the second conservation law in (4.41) is another Casimir (see also Ref. [42]). However, it follows directly from [42] that the Casimir invariants are related to the symmetries of the Lagrangian representation.
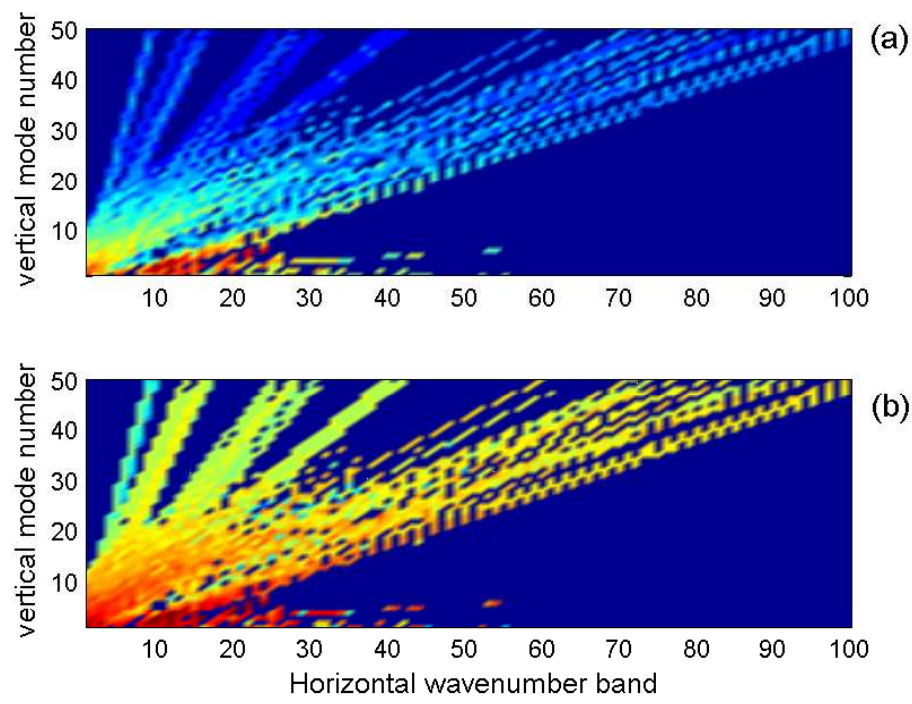

FIgURE 1. Beam-like structure of the GM energy distribution in wavenumber space for 20000 resonantly interacting waves in a slow interaction time scale. Panel (a) shows the energy distribution at initial time and panel (b) shows the energy distribution at a latter time of 50 inertial periods.

For visualization purposes, Figure 1 illustrates the results of numerical simulations for energy distribution in the wavenumber space between 20000 resonantly interacting internal waves at latitude $20^{\circ}$ North ([24]). The numerical model used in ([24]) is based on the governing equations (4.24) - (4.26) and represents the resonant triad simulator designed to study the energy exchange between arbitrarily large number of coupled resonant waves with frequencies spanning the range of possible frequencies, i.e., between a maximum of the buoyancy frequency and a minimum of the inertial frequency (two limiting cases of internal wave propagation) and mode numbers between $n_{\min }=1$ and $n_{\max }=50$. The similarity with atmospheric waves in a thin spherical shell has also been considered in our recent studies in [26]. As an illustration of the simulations shown in Figure 1, the averaged initial energy density of each wave in the model is given by the energy density in the conservation law (4.41), $v_{i}^{2}+g^{2} \rho_{i}^{2} / N^{2}+\left|\nabla \psi_{i}\right|^{2}$, where $i=1, \ldots, 20000$ is a wave number. The initial wave amplitudes were initialized on the the choice of the 


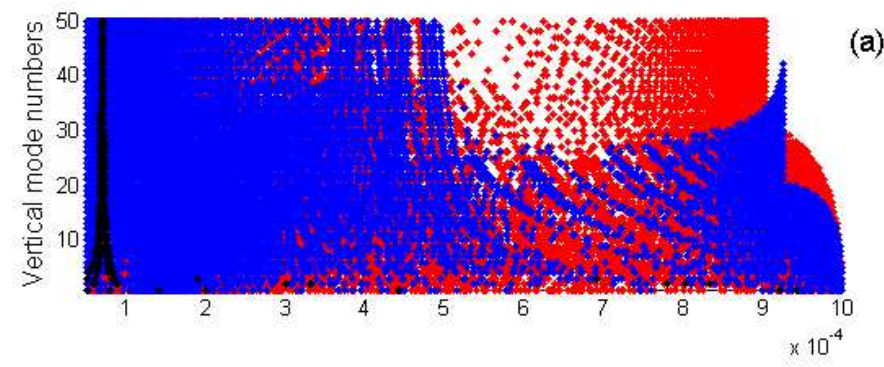

(a)

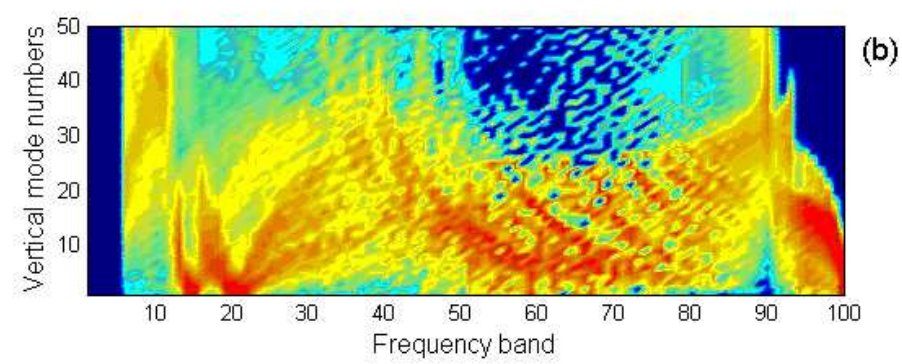

Figure 2. Panel (a) shows the resonant wave distribution in $(\omega, n)$-space. Cayan points are used to indicate waves generated resonantly from the primary wave (Level 0 waves), blue and red points represent Level 1 and level 2 waves generated from all the Level 0 and level 1 waves (see [24] for the nomenclature). Panel (b) shows the corresponding initial energy distribution.

Garrett and Munk (GM) spectrum [9]. The GM spectrum is often used as a representative statistical description of the internal wave field in studies of nonlinear wave interactions and mixing parameterization. Observations of the internal wave spectrum in the deep ocean indicate the remarkable fact that it has a very similar shape wherever it is observed, unless the observations are made close to a strong source of internal waves. A fundamental role in spanning the universal deep ocean spectrum could be attributed to weakly nonlinear-wave interactions within the internal wave field which smooth out any spectral irregularity by redistributing energy within the spectrum. Thus, the GM spectrum provides an important link in the overall energy cascade from the large generation scale to the small dissipation scale.

It is of interest to note that the energy distribution in wavenumber space shown in Figure1 has a beam-like structure which is strongly affected by the effects of the earth's rotation.

The corresponding wave and energy distribution in the frequency - vertical (discrete mode) number space is shown in Figure 2. The interested reader is addressed to the reference [24] for more details on the resonant triad simulator.

\section{Concluding Discussion}

The emphasis of this review has been on applications of Lie group analysis of differential equations to internal waves in the ocean. This approach represents an alternative prospect of using of using completely-integrable equations of Hamiltonian type internal waves [2], [30], [31]. One of the advantages of Lie group analysis consists in the fact that it can be used to integrate nonlinear equations that are not of Hamiltonian type. As a particular example, it is demonstrated in this paper, that the invariant $\eta=k x+m z$ of the translation symmetry provides the class of exact solutions that were deduced in the previous studies from the anisotropic nature of internal wave propagation (see e.g., [45], [44], [5] and [34]). However, the more general forms of invariant solutions, which can not be guessed from the 
anisotropic property and correspondingly were not reported in previous studies, are presented by the infinite-dimensional Lie algebra spanned by the infinitesimal symmetries (4.29) - (4.33). Preliminary Lie group analysis of the rotationally invariant solution (4.34) - (4.36) for internal gravity waves in the ocean, namely the analytic and numerical comparison with the linear approximation and experimental results on internal wave beams generated from a vertically oscillating sphere studied in [7], can also be found in our pre-prints [21]. The more detailed analysis on the subject will appear elsewhere.

There are some limitations with the approach in the present study. First, the present simulations were restricted to two-dimensional internal wave motions so that the present model lacks interaction of internal waves with different types of motion such as vortical modes [40]. Second, the stratification considered here is highly idealized, having constant buoyancy frequency which is commonly used in laboratory and theoretical experiments but it is not common in the ocean with the exception when considering short wavelengths in comparisons with the scale of density changes ([43]). These compromise the quantitative application of our results to the real ocean.

\section{References}

[1] H. Cho, T. Shepherd, V. Vladimirov. Application of the direct Liapunov method to the problem of symmetric stability in the atmosphere. J. Atmosph. Sci., (1993), 50 (6), 822-836.

[2] W. Craig, P. Guyenne, H. Kalisch. Hamiltonian long-wave expansions for the free surfaces and interfaces. Comm. Pure Appl. Math., (2005), 58, 1587-1641.

[3] E. Dewan, R. Picard, R. O'Neil, H. Gardiner, J. Gibson. MSX satellite observations of thunderstorm-generated gravity waves in mid-wave infrared images of the upper stratosphere. Geophys. Res. Lett., (1998), 25, 939-942.

[4] S. Dalziel, G. Hughes, B. Sutherland. Whole field density measurements by synthetic schlieren. Experiments in Fluids, (2000), 28, 322-337.

[5] T. Dauxois, W. Young. Near-critical reflection of internal waves. J. Fluid Mech., (1999), 390, 271-295.

[6] R. Fjortoft. R, Application of integral theorems in deriving criteria of stability for laminar flows and for the baroclinic circular vortex. Geophys. Publ., (1950), 17(6), 1-52.

[7] M. Flynn, K. Onu, B. Sutherland. Internal wave excitation by a vertically oscillating sphere. J. Fluid Mech., (2003), 494, 65-93.

[8] C. Garrett. Internal tides and ocean mixing. Science, (2003), 301 (5641), 1858-1859, doi:10.1126/science.1090002.

[9] C. Garrett, W. Munk. Space time scale of internal waves. A progress report. J. Geophys. Res., (1975), 80, $291-297$.

[10] A. Gill. Atmosphere-Ocean Dynamics. New York, etc., Academic Press, (1983).

[11] J. Hadamard. Lectures on Cauchy's problem in linear partial differential equations. Yale University Press, New Haven, (1983).

[12] J. Hadamard. The problem of diffusion of waves. Annals of Mathematics, Ser. 2 43: 510-522, (1942).

[13] N. Ibragimov. Elementary Lie group analysis of ordinary differential equations. John Wiley \& Sons, Chichester, (1999).

[14] N. Ibragimov. Transformation Groups Applied to Mathematical Physics. Nauka, Moscow (1983), English. transl., Reidel, Dordrecht.

[15] N. Ibragimov Ed. CRC Handbook of Lie group analysis of differential equations (CRC Press, Boca Raton); Vol. 1 (1994, 429 p), Vol. 2 (1995, 546 p.), Vol. 3 (1996, 536 p.).

[16] N. Ibragimov. A new conservation theorem J. Math. Anal. Appl., (2007), 333: 311-328.

[17] N. Ibragimov. Group analysis - a microscope of mathematical modelling. I: Galilean relativity in diffusion models. Selected works (ALGA Publications, Karlskrona), (2006), 2: 225-243.

[18] N. Ibragimov. Conformal invariance and Huygens' principle. Soviet Mathematics Doklady, (1970), 11(5): $1153-1157$.

[19] N. Ibragimov. Application of group analysis to liquid metal systems. Archives of ALGA, (2010), 6: 91-101.

[20] N. Ibragimov. Lie group analysis of Moffatt's model in metallurgical industry. Nonlinear Math. Phys., (2011), 18, 143-162.

[21] N. Ibragimov, R. Ibragimov, V. Kovalev. Group analysis of nonlinear internal waves in oceans. Archives of ALGA, (2009), 6, 45-54.

[22] N. Ibragimov, R. Ibragimov. Applications of Lie Group Analysis in Geophysical Fluid Dynamics. Series on Complexity, Nonlinearity and Chaos, (2011), Vol 2, World Scientific Publishers, ISBN: 978-981-4340-46-5.

[23] N. Ibragimov, R. Ibragimov. Internal gravity wave beams as invariant solutions of Boussinesq equations in geophysical fluid dynamics. Comm. Nonlinear Sci. Num. Simulat., (2010), 15, 1989-2002.

[24] R. Ibragimov. Oscillatory nature and dissipation of the internal wave energy spectrum in the deep ocean. Eur. Phys. J. Appl. Phys., (2007), 40, 315-334.

[25] R. Ibragimov. Generation of internal tides by an oscillating background flow along a corrugated slope. Phys. Scr. , (2008), 78, 065801.

[26] R. Ibragimov, D. Pelinovsky. Incompressible viscous fluid flows in a thin spherical shell. J. Math. Fluid. Mech., (2009), $11,60-90$. 
[27] R. Ibragimov, N. Ibragimov. Effects of rotation on self-resonant internal gravity waves. Ocean Modelling, (2010), 31, 80-87.

[28] R. Ibragimov, M. Dameron. Spinning phenomena and energetics of spherically pulsating patterns in stratified fluids. Phys. Scr., (2011), 84, 015402.

[29] A. Javam, J. Imberger, S. Armfield. Numerical study of internal wave-wave interactions in a stratified fluid. J. Fluid Mech., (2000), 415, 65-87.

[30] H. Kalisch, N. Nguyen. On the stability of internal waves. J. Phys. A, (2010), 43, 495205.

[31] H. Kalisch, J. Bona. Modes for internal waves in deep water. Disc. Cont. Dyn. Sys. (2000), 6, 1-19.

[32] A. Kistovich, Y. Chashechkin. Nonlinear interactions of two dimensional packets of monochromatic internal waves. Izv. Atmos. Ocean. Phys., (1991), 27 (12) 946-951.

[33] F. Lam, L. Mass, T. Gerkema. Spatial structure of tidal and residual currents as observed over the shelf break in the Bay of Biscay. Deep-See Res., (2004), I 51, 10751096.

[34] P. Lombard, J. Riley. On the breakdown into turbulence of propagating internal waves. Dyn. Atmos. Oceans, (1996), 23, 345-355.

[35] H. Moffatt. High frequency excitation of liquid metal systems. Metallurgical Applications of Magnetohydrodynamics, (1984), (Metals Society, London) 180-189.

[36] P. Müller, G. Holloway, F. Henyey, N. Pomphrey. Nonlinear interactions among internal gravity waves. Rev. Geophys., (1986), 24, 3, 493-536.

[37] J. Nash, E. Kunze, C. Lee, T. Sanford. Structure of the baroclinic tide generated at Keana Ridge, Hawaii. J. Phys. Oceanogr., (2006), 36, 1123-1135.

[38] P. Olver. Applications of Lie groups to differential equations. Springer-Verlag, New York, 2nd ed. 1993.

[39] L. Ovsyannikov. Group Analysis of Differential Equations. Nauka, Moscow, (1978), English transl., ed. W.F. Ames, Academic Press, New York (1982).

[40] D. Ramsden, G. Holloway. Energy transfers across an internal wave-vortical mode spectrum. J. Geophys. Res., (1992), 97, 3659-3668.

[41] J.Riley, R. Metcalfe, M. Weissman. Direct numerical simulations of homogeneous turbulence in density-stratified fluids. Nonlinear properties of internal waves, (1981), 76, edited by B.J. West, pp. 79-112, Americal Institute of Physics, New York.

[42] T. Shepherd. Symmetries, conservation laws, and Hamiltonian structure in geophysical fluid dynamics. Advances in Geophysics, (1990), 32, 287-338

[43] C. Staquet, J. Sommeria. Internal Gravity Waves: From instabilities to turbulence. Annu. Rev. Fluid Mech., (2002), 34, 559-593.

[44] A. Tabaei, T. Akylas, K. Lamb. Nonlinear effects in reflecting and colliding internal wave beams. J. Fluid Mech., (2005), 526, 217-243.

[45] A. Tabaei, T. Akylas. Nonlinear internal gravity wave beams. J. Fluid Mech., (2003), 482, 141-161.

[46] S. Teoh, J. Imberger, G. Ivey. Laboratory study of the interactions between two internal wave rays. J. Fluid Mech., (1997), 336:91.

[47] K. Winters, E. D'Asaro. Direct simulation of internal wave energy transfer. J. Phys. Oceanogr., (1997), 9, $235-243$.

[48] C. Wunsch, R. Ferrari. Vertical mixing, energy, and the general circulation of the oceans. Annu. Rev. Fluid Mech., (2004), 36, 281-314.

[49] H. Zhang, B. King, H. Swinney. Resonant generation of internal waves on a model continental slope. Phys. Rev. Let., (2008), 100, 244504. 